\title{
The 2009/10 Drought in China: Possible Causes and Impacts on Vegetation
}

\author{
DAVID BARRIOPEDRO \\ IDL, Universidade de Lisboa, Lisbon, Portugal, and Departamento de Astrofísica y Ciencias de la Atmósfera/Instituto de \\ Geociencias, Universidad Complutense de Madrid, Madrid, Spain \\ CÉlia M. Gouveia \\ IDL, Universidade de Lisboa, Lisbon, and Escola Superior de Tecnologia, Instituto Politécnico de Setúbal, Setubal, Portugal \\ RICARDO M. TRIGO \\ IDL, Universidade de Lisboa, and Departamento de Engenharias, Universidade Lusófona, Lisbon, Portugal \\ LIN WANG \\ Center for Monsoon System Research, Institute of Atmospheric Physics, Chinese Academy of Sciences, Beijing, China
}

(Manuscript received 22 June 2011, in final form 16 February 2012)

\begin{abstract}
Several provinces of China experienced an intense drought episode during 2009 and 2010. The drought was particularly severe in southwestern and northern China, where the accumulated precipitation from May 2009 to April 2010 was about $25 \%$ less than normal. The decline of accumulated precipitation over northern China was mostly noticeable during the summer season of 2009 and it was comparable to recent dry episodes. The southwestern China drought resulted from a sequence of dry months from summer 2009 to winter 2010 , corresponding to the driest event since at least 1951. The suppression of rainfall in summer over both regions was in agreement with a weakened broad-scale South Asian summer monsoon, possibly influenced by an El Niño developing phase, whereas the extremely negative phases of the Arctic Oscillation during the winter of 2010 may have contributed to the persistence of the drought in southwestern China. The assessment of the associated impacts indicates that water reservoirs were severely affected with a $\sim 20 \%$ reduction in the nationwide hydroelectrical production during the drought event. Furthermore, an analysis of the normalized difference vegetation index data reveals that large cropland sectors of northern and eastern China experienced up to 8 months of persistently stressed vegetation between May 2009 and July 2010, while southwestern China was relatively less affected. Such different regional vegetative responses are interpreted in terms of the land-cover type, agriculture management, and their dependence on seasonal precipitation and water availability for irrigation.
\end{abstract}

\section{Introduction}

During 2009 and 2010, an intense and prolonged drought episode affected several provinces of northern (Hebei, Shanxi, and Liaoning) and southwestern (Yunnan, Guizhou, Guangxi, and Sichuan) China, including the municipalities of Beijing and Chongqing (Qiu 2010). Northern China is an important area of grain

Corresponding author address: David Barriopedro, Instituto Dom Luiz, Universidade de Lisboa, Departamento de Engenharia Geográfica, Geofísica e Energia, Faculdade de Ciências, Univ. de Lisboa, Campo Grande, Ed C8, Piso 6, 1749-016 Lisboa, Portugal. E-mail: dbarriopedro@fc.ul.pt production, while the southwest region represents one of the major water resources of the country, supplying water to the Yangtze River, the Pearl River head, and their reservoirs. According to the Chinese Office of State Flood Control and Drought Relief Headquarters and the Ministry of Civil Affairs, the drought affected $\sim 25$ million people and $\sim 18$ million livestock by shortage of drinking water, as well as about $\sim 8$ million ha of arable land nationwide, with total economic losses of at least $\$ 3.5$ billion U.S. dollars (http://www.chinadaily.com.cn/ china/2010drought/index.html).

Droughts are recurrent phenomena in China, but they can be associated to different causes and complex 
processes. For example, the recently severe drought episode during winter 2008/09 in eastern China was shown to be partially driven by the $2008 / 09$ La Niña episode and also influenced by the anomalous high temperatures over the Tibetan Plateau (Gao and Yang 2009). Interannual rainfall variations in China are also associated with the Asian monsoon-a complex and multifaceted system encompassing the East Asian monsoon (EAM), the Indian monsoon (IM), and the western North Pacific monsoon (WNPM) (e.g., Wang et al. 2001; Wang and LinHo 2002). The EAM (e.g., Ding and Chan 2005) is characterized by a cold, dry East Asian winter monsoon (EAWM) affecting Japan and southeastern China (e.g., Zhang et al. 1997) and a warm, rainy East Asian summer monsoon (EASM), whose main signature is a meridional rainfall tripole and the quasi-stationary mei-yu subtropical front (e.g., Chen et al. 2004). Fluctuations in the EASM are often associated with floods and droughts in the Yangtze River basin (e.g., Huang et al. 2007). Relatively, other regions of the country are more affected by interactions between the different subsystems of the Asian monsoon, midlatitude processes, and tropical cyclones (e.g., Wang et al. 2008), which in turn are subject to remote influences. In particular, El Niño-Southern Oscillation (ENSO) explains a significant part of the interannual Asian monsoon variability by displacing the heat sources in the tropics (Webster and Yang 1992; Wang et al. 2001), although such relationship is nonstationary (Kumar et al. 1999; $\mathrm{R}$. Wu and B. Wang 2002). Other reported factors affecting seasonal precipitation in China are the Arctic Oscillation/North Atlantic Oscillation (AO/NAO; Gong and Wang 2003; Sung et al. 2006), the stationary planetary waves (Chen et al. 2005), the Antarctic Oscillation (AAO; Nan and Li 2003), dynamic and thermal effects of the Tibetan Plateau (Wu and Zhang 1998; Hsu and Liu 2003), and the Eurasian snow cover (Zhang et al. 2004).

In addition to the assessment of physical mechanisms associated with extreme events, it is of equal relevance to quantify their impacts. This is a particularly daunting task in China, where the area influenced by droughts reaches nearly 20 million $\mathrm{km}^{2}$ on average every year, accounting for about $1 / 6$ of the national arable land (Huang et al. 2000). Given the key role of agriculture ( $\sim 13 \%$ of China's GDP) sustaining the huge number of Chinese people ( $\sim 1.3$ billion), droughts can cause large economic losses in China (circa $50 \%$ of the total losses induced by climatic hazards on average; Huang et al. 2000). With the advent of satellite-derived products, the monitoring of droughts and the related assessment of vegetation health and net primary production have experienced significant improvements (e.g., Kogan 1995; Fang et al. 2003). Studies based on the normalized difference vegetation index (NVDI) over China have shown a nationwide enhancement of vegetation coverage during recent decades, which is not only due to the advance of agricultural technology but also to the regional precipitation and temperature changes that have caused an extended growing season and more rapid plant growth (Piao et al. 2010). This general trend in NDVI is, however, punctuated by severe droughts with spatially coherent NVDI anomaly patterns (Habib et al. 2009), which have become relatively frequent and widespread in northern China since the 1970s (e.g., Zou et al. 2005). The application of NDVI as a tool to assess droughts' impacts in agriculture production is also of great interest in China because the large variety of climate regimes and crops, together with an uneven distribution of water resources and agriculture management, make that a given combination of climate anomalies can produce either beneficial, irrelevant, or damaging effects on vegetation (e.g., Tao et al. 2008).

The main goal of this paper is to provide a description of the 2009/10 drought in China from multiple perspectives by addressing some of the aforementioned issues. In more detail, the objectives are (i) to characterize the temporal and spatial extent of this extreme drought event, (ii) to assess the regional precipitation deficits in terms of the associated atmospheric circulation anomalies, and (iii) to evaluate some socioeconomic impacts of this extreme drought episode, particularly on vegetation greenness and hydroelectrical production.

The data and methods employed in this study are described in section 2. Section 3 analyzes the spatial and temporal characteristics of this drought. The associated anomalous atmospheric circulation is presented in section 4. Section 5 then describes the social-economic impacts of this drought, including the impacts on national hydroelectrical production and on vegetation. Finally, the main conclusions are presented in section 6 .

\section{Data and methods}

Monthly precipitation data on land surface at $1.0^{\circ} \times$ $1.0^{\circ}$ resolution grid were provided by the Global Precipitation Climatology Centre (GPCC; Rudolf and Schneider 2005). This dataset has already been used to analyze extreme dry precipitation episodes over different regions, such as Iberia (García-Herrera et al. 2007) and the Middle East (Trigo et al. 2010). The GPCC products are based on in situ rain gauge data and cover the period from 1901 to present through two gridded datasets: the so-called full data product (1901-2009), which is continuously updated with all available stations, and the monitoring product (2007-present) that only uses a network of near-real-time stations. Both datasets are subject to 


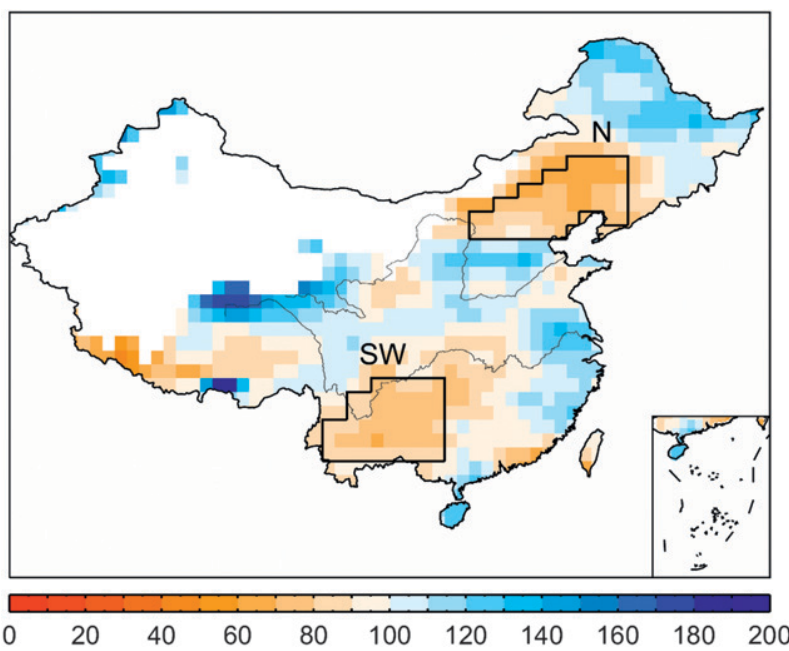

FIG. 1. Spatial distribution of the accumulated monthly precipitation (in percentage relative to the 1951-2008 norm) during the hydrological year 2009/10 (i.e., between May 2009 and April 2010). To avoid misleading results over dry areas, only grid points with climatological accumulated precipitation above $240 \mathrm{~mm}$ (i.e., $20 \mathrm{~mm}$ month $^{-1}$ ) are shown. Black boxes approximately delimit regions with maximum deficit in accumulated precipitation and they are referred to as $\mathrm{N}$ and SW China in the text.

similar processing and quality-control steps but they differ in the number of underlying stations. A preliminary analysis revealed high consistence between these two GPCC products during their overlapping period (200709). Therefore, the monitoring product for 2010 was added to the full data product (available until the end of 2009) to provide full temporal coverage of the drought episode (2009/10). Since the number of stations over China experiences a significant decrease before the 1950s, the period of analysis has been limited to 1951-2010. To better characterize regional features of the drought episode, regional precipitation averages have been computed over northern $(\mathrm{N})$ and southwestern (SW) China (see Fig. 1) after excluding grid points with very dry climates (e.g., inner Mongolia) and/or at high elevation sites (e.g., southeasternmost sector of the Tibetan Plateau). The spatial domain of these sectors is further supported by a clustering analysis based on observational precipitation stations in China (Song et al. 2007).

The atmospheric data include geopotential height, temperature, humidity, and zonal and meridional wind data at different pressure levels obtained from the National Centers for Environmental Prediction-National Center for Atmospheric Research (NCEP-NCAR) reanalysis in a $2.5^{\circ} \times 2.5^{\circ}$ regular grids and for the period 1951-2010 (Kistler et al. 2001). The vertically integrated moisture flux (from the surface to $300 \mathrm{hPa}$ ) was computed following the approach adopted in Trenberth and Guillemot (1995). This field displayed almost identical features to the water vapor flux at low levels (e.g., $850 \mathrm{hPa}$ ) for the analyzed period of the drought and it also showed strong resemblance with the low-level wind, except over relatively dry land areas of midlatitudes. With the aim of assessing remote influences in the regional precipitation variability, we have used atmospheric circulation indices of ENSO [measured by the El Niño 3.4 index (EN3.4)] and the AO for the 1951-2010 period, as provided by the Climate Prediction Center of the National Oceanic and Atmospheric Administration (NOAA; www. cpc.ncep.noaa.gov/).

Additionally, taking into account the regional manifestations of the Asian monsoon, a suite of distinct winter and summer monsoon indices have been computed from the NCEP-NCAR reanalysis for the period 19512010. The summer indices include (i) the Webster-Yang Asian Monsoon Index (WYMI; Webster and Yang 1992), (ii) the Western North Pacific Summer Monsoon Index (WNPMI; Wang and Fan 1999), and (iii) the Indian Monsoon Index (IMI; Wang and Fan 1999). In what concerns the winter season, the strength of the EAWM was characterized by the East Asian Winter Monsoon Index (EAWMI) proposed by Jhun and Lee (2004). Seasonal values are computed from the seasonal mean fields and then standardized with reference to the 19512008 period. The WYMI reflects the broad-scale South Asian summer monsoon variability, which is primarily driven by two relatively independent convective heat sources over the Bay of Bengal and the Philippine Sea (Wang and Fan 1999), herein represented by the IMI and WNPMI, respectively. Although the WNPM is not exactly the same system as EASM, it is closely related to the variations of mei-yu and therefore is able to capture the main features of the EASM (Wang et al. 2008). The sign of WNPMI used here is reversed compared with the original index proposed by Wang and Fan (1999) so that positive value indicates an enhanced mei-yu rainfall. Other summer and winter monsoon indices (see Wang et al. 2008 and Wang and Chen 2010a) may capture better regional precipitation anomalies, but for the sake of simplicity, the discussion will focus on some of the most traditional indices that are of standard use in operational centers.

The response of vegetation was assessed with fields of NDVI since a close relationship between NDVI and annual rainfall variations has been reported for different land-cover types in China (Fang et al. 2001). Meanwhile, it should be noted that water intensive crops (e.g., rice and cotton) and areas with multicropping rotations are more sensitive to seasonal rains, springtime snow, and glacier melt and water resources (Piao et al. 2010). The NDVI can also reflect temperature anomalies since low minimum temperatures during the growing season cause 
damage to crops (e.g., Tao et al. 2008). NDVI values were derived from the so-called 10-day global synthesis (S10) products of the SPOT-VEGETATION partner (VITO) database (http://free.vgt.vito.be), which provide atmospherically corrected and geometrically calibrated data acquire by the VEGETATION instrument on board both Satellite Pour l'Observation de la Terre (SPOT) SPOT-4 and SPOT-5 satellites between September 1998 and August 2010 (Maisongrande et al. 2004; Hagolle et al. 2005). NDVI fields are supplied at the resolution of $0.008928^{\circ}$ (i.e., about $1-\mathrm{km}^{2}$ resolution at the equator) in geographic coordinates and on a 10-day basis, following the maximum value composite (MVC) method, which selects the date of maximum NDVI among 10 consecutive daily images (Holben 1986). All months are divided in three 10-day values, the first one corresponding always to the first 10-day period of that month. For months with 31 days and February ( 28 or 29 days), the last 10day value actually represents the maximum NDVI within slightly longer (11 day) or shorter ( 8 or 9 day) periods. Monthly fields of the NDVI were subsequently derived by averaging at each pixel the three 10-day values of the given month.

It should be noticed that NDVI values tend to underestimate the green biomass of stands over areas with strong foliage density and high production (Hobbs 1995; Tucker et al. 1986; Gilabert et al. 1996), causing saturation of NDVI. Despite this caveat, which is expected to cause relatively lower impacts in the assessment of droughts, the time series of MVC-NDVI composites have proven to be a source of valuable information for monitoring surface vegetation dynamics at the global and the regional scales (Zhou et al. 2001; Nemani et al. 2003; Gouveia et al. 2008). Therefore, despite the relatively short period analyzed herein, the use of this dataset is preferred because the high spatial resolution is expected to bring a more accurate assessment of the drought impacts in land-cover types. Information about the land-cover type associated to each pixel was obtained from the Global Land Cover 2000 database (http://bioval. jrc.ec.europa.eu/products/glc2000/products.php).

\section{Spatial and temporal characteristics of the drought}

The precipitation regime in China is characterized by a strong seasonal behavior, with a rainy season mostly concentrated between May to September and a large spatial gradient in the annual totals between the wet monsoonal areas of the southeast and the semiarid regime of northwest (e.g., Song et al. 2007). Therefore, the hydrological year used in this study is defined as the period spanning between May of year $(y r)$ and April of the next year $(y r+1)$. Meanwhile, the amount and timing of the annual maximum of monthly precipitation varies across China following the penetration and northwestern advance of the Asian monsoonal flows. Thus, SW China experiences a long-lasting and intense summer monsoon, with a broad precipitation peak of $\sim 200 \mathrm{~mm} \mathrm{month}^{-1}$ centered in June-July that contrasts with the relatively narrower, delayed (July-August), and less intense $\left(150 \mathrm{~mm}\right.$ month $\left.^{-1}\right)$ maximum of $\mathrm{N}$ China (see Figs. 2b,c). However, when annual totals are considered N China shows a stronger dependence on summer rainfall, with July-August precipitation accounting for more than half of the annual totals. This implies that dry summer conditions in $\mathrm{N}$ China are expected to be reflected on the accumulated totals, while a dry summer monsoon in SW can be compensated by near-normal precipitation in other seasons.

To better assess the drought episode, the spatial pattern of accumulated precipitation percentages for the hydrological year (May 2009-April 2010) has been computed with respect to the corresponding climatological (1951-2008) norms (Fig. 1). The drought episode was particularly severe over N and SW China, with both regions recording nearly $25 \%$ below the climatological normal. In addition, relatively less severe drought conditions were observed in other territories, including northeastern India and Burma; northern parts of Thailand, Laos, and Vietnam (not shown); as well as other provinces of southern China such as Hunan and Guangdong (Fig. 1).

Figure 2a shows the accumulated monthly precipitation averaged over N and SW areas between May 2009 and April 2010, along with the corresponding evolution of the climatological accumulated monthly precipitation distribution (whiskers plot). At the end of the considered period, the regional average accumulated precipitation in SW $(\sim 850 \mathrm{~mm})$ was $\sim 25 \%$ less than the long-term mean value $(\sim 1100 \mathrm{~mm})$, corresponding to the lowest accumulated value in any hydrological year since at least 1951. In relative terms, the $\mathrm{N}$ region experienced a more severe decrease $(\sim 30 \%)$ in accumulated totals $(\sim 350 \mathrm{~mm}$ relative to a $\sim 470 \mathrm{~mm}$ mean $)$. However, this region is also characterized by large interannual variability, with the standard deviation (SD) of annual accumulated totals $(69.7 \mathrm{~mm})$ being of similar magnitude to that in SW $(76.2 \mathrm{~mm})$. Consequently, from a normalized point of view, the 2009/10 drought was more severe in SW $(-3.1 \mathrm{SDs})$ than in $\mathrm{N}(-1.7 \mathrm{SDs})$. It is worth noticing that, despite its extreme magnitude, the 2009/10 drought episode over N China was not particularly anomalous in the context of recent years (Fig. 2a), which have been characterized by a relative recurrence of extreme droughts. This is in agreement 
a)

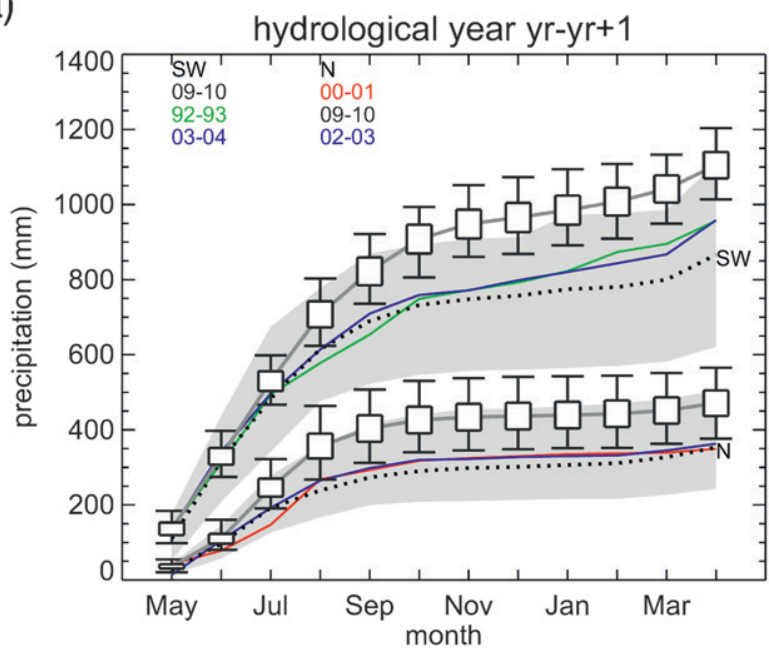

b)

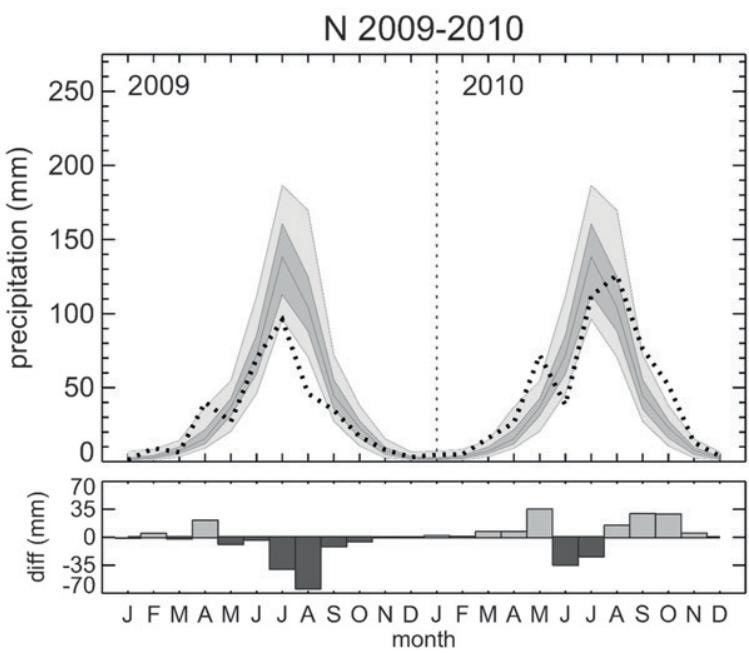

C)

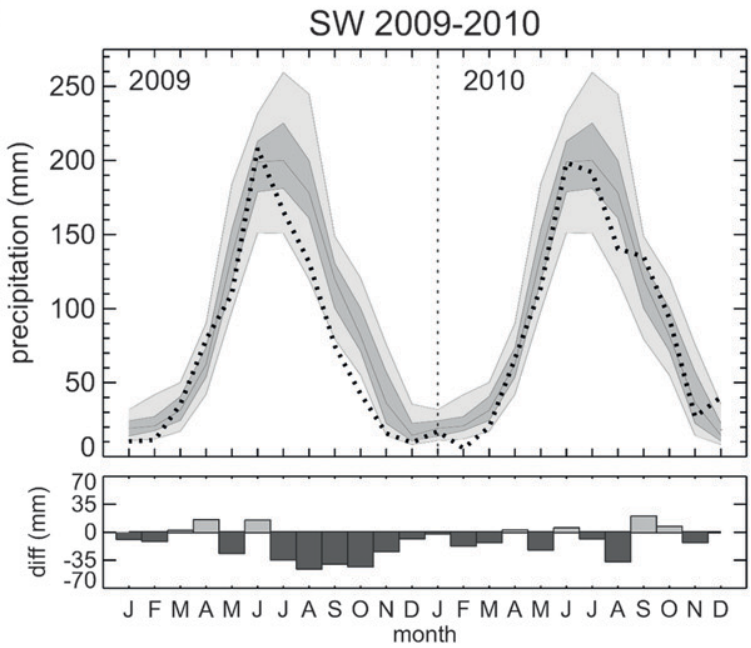

FIG. 2. (a) Accumulated monthly precipitation averaged over N and SW China during the hydrological year 2009/10 (black dotted lines). Gray shaded areas show the overlapping from the corresponding evolution from all grid points embedded in the given area with a drying climate trend in northern China (e.g., $\mathrm{Hu}$ et al. 2003) and the increasing tendency for $\mathrm{N}$ China to suffer from severe and long-lasting droughts, which were particularly acute from $1997 / 98$ to 2002/03 (Zou et al. 2005; Wang et al. 2011).

To better assess the months that specifically contributed to the marked decline in accumulated precipitation within the hydrological year, Figs. $2 b$ and $2 c$ show the temporal evolution of the regional averaged precipitation monthly series during 2009 and 2010 in N and SW China, respectively. The period with deficit of precipitation started in May 2009 and lasted until October 2009 in N China, further persisting until March 2010 in SW China. The winter and spring of 2010 were also dry in the middle and the lower reaches of the Yellow and Yangtze Rivers (not shown), but slightly wet in N China (Fig. 2b). A near-normal 2010 monsoon season in SW (N) China contributed to alleviate (mitigate) the persistent deficits accumulated throughout the previous hydrological year.

Compared with the historical situation, every month of the May-September monsoon period of 2009 (except June) exhibited precipitation deficits below the 30th percentile of the long-term distribution in both $\mathrm{N}$ and SW China (Figs. 2b,c). However, the drought in these two areas exhibits different features. Thus, in relative terms, the precipitation deficit over SW China was more severe (below the 10th percentile of its climatological distribution) from September to November 2009 (i.e., after the peak time of local monsoon rainfall). In contrast, $\mathrm{N}$ China received the lowest precipitation (also below the monthly 10th percentile) during JulySeptember 2009, which is the peak time of local monsoon rainfall. As a consequence, the drought period over $\mathrm{N}$ China was shorter and mainly caused by decreased summer precipitation, whereas that over the relatively wetter SW China resulted from the persistence of dry conditions during several consecutive months.

(defined by the black boxes of Fig. 1). Gray lines indicate the climatological mean evolution in each region, with boxes (whiskers) representing the \pm 0.5 -sigma level (10th-90th percentiles) obtained from all hydrological years between 1951 and 2008. The other two most severe drought events in each region within the period 19512008 are also indicated with color lines and ranked in the upper-left corner. (b),(c) Climatological (1951-2008) annual cycle of monthly precipitation averaged over (b) $\mathrm{N}$ and (c) SW China. Two full annual cycles are shown. The light (dark) shaded areas comprise the 10th-90th (30th-70th) monthly percentiles obtained from the 1951-2008 precipitation time series, with the median in between. Dashed line shows the time series for 2009 and 2010, with the corresponding monthly departure from the climatological mean being represented in the bottom graphic. 
a)

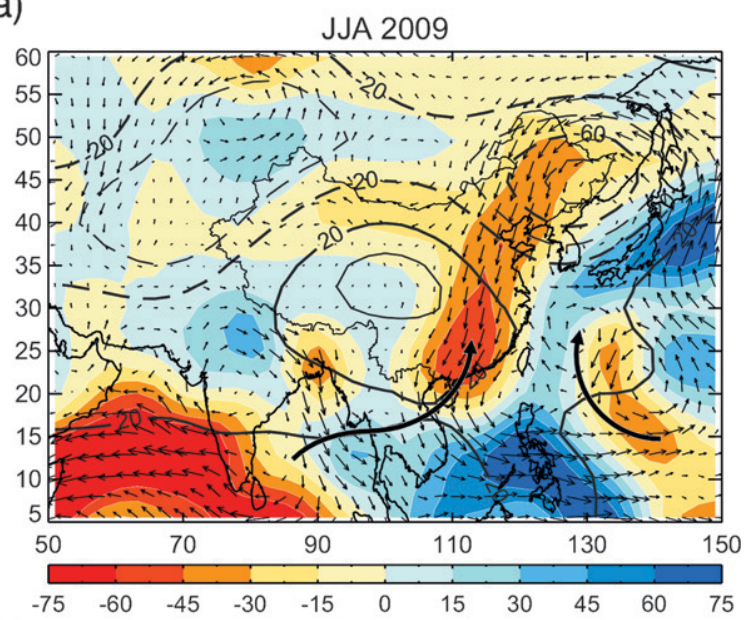

b)

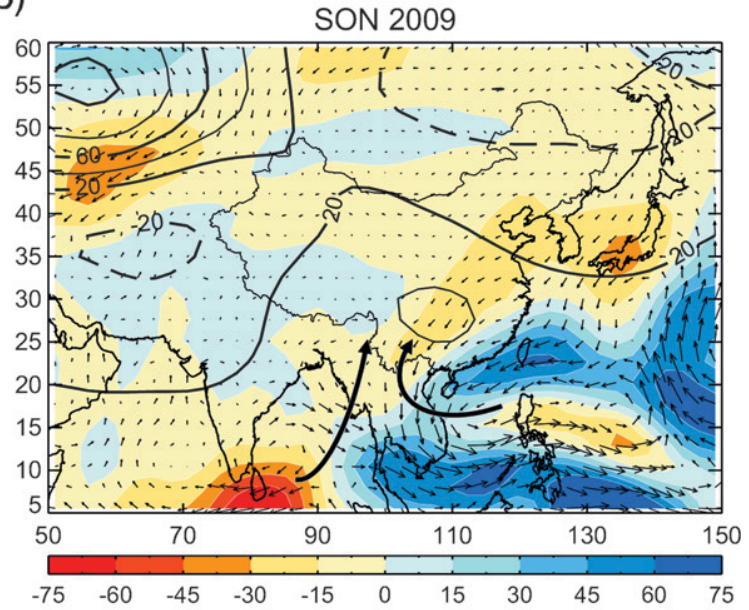

c)

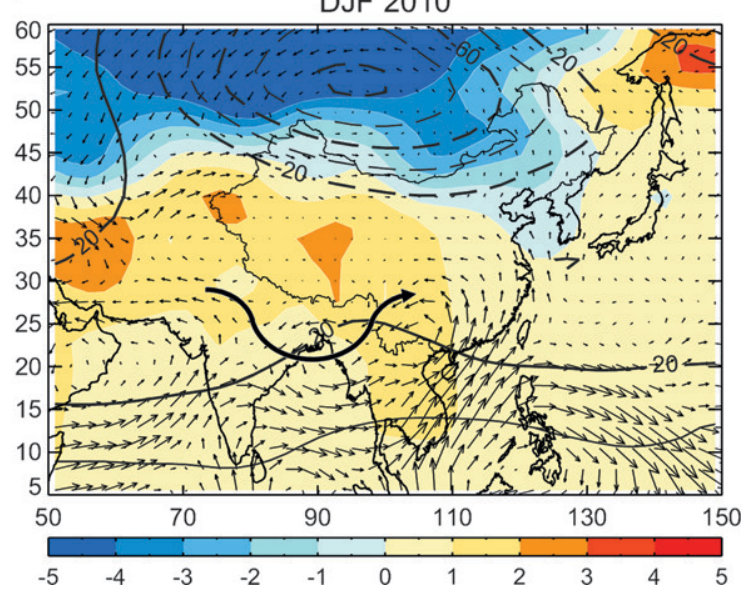

FIG. 3. Seasonal anomalies of geopotential height at $200 \mathrm{hPa}$ (gpm, contours), vertically integrated moisture fluxes from surface to $300 \mathrm{hPa}\left(\mathrm{kg} \mathrm{m}^{-1} \mathrm{~s}^{-1}\right.$, arrows), and total water transport ( $\mathrm{kg} \mathrm{m}^{-1} \mathrm{~s}^{-1}$, shading) for (a) summer 2009, (b) autumn 2009, and (c) as in (a) but for winter 2010, and with shading indicating surface temperature anomalies $\left({ }^{\circ} \mathrm{C}\right)$. Black thick arrows indicate the main climatological channels of water flux transport over the areas affected by the drought at the given season. Anomalies are relative to the 1951-2008 period.

\section{Atmospheric circulation during the drought episode}

\section{a. Some possible causes of the drought}

This section describes briefly the large-scale features that characterized the drought episode over China and enumerates some of the factors with impact in $\mathrm{N}$ and SW China precipitation. The analyses aim at identifying potential candidates that might have contributed to the drought episode. A definitive cause-effect attribution would require a full screening process of all reported physical mechanisms with precipitation impacts over the affected areas and also the use of a modeling component. It follows that the described factors most likely correspond to a subset of all possible causes for the severity of the drought. For the sake of simplicity, we restrict the analysis to the three seasons that experienced stronger regional precipitation deficits-namely, summer [June-August (JJA)] of 2009 (in N and SW China), autumn [September-November (SON)] of 2009 (SW), and winter [December-March (DJFM)] of 2009/10 (SW), referred to as winter of 2010.

An inspection of climatological water fluxes indicates that the main channel of transporting moisture into SW China mostly results from the convergence and posterior turn to southerlies of the westerly winds from the Indian Ocean and the easterlies induced by the western North Pacific subtropical high (thick arrows Fig. 3), although with varying contribution of these two components through the course of the extended summer (e.g., Ju et al. 2006). The summer monsoon season of 2009 was characterized by a height dipole at the lower troposphere (e.g., $850 \mathrm{hPa}$ ) with higher-than-normal pressures centered over northern China and Mongolia and lower-than-normal pressures over the western Pacific (not shown). As a consequence, the climatological northwest-southeast land-sea pressure contrast was reduced, weakening the low-level southerlies along a corridor stretching from southern to northern China. This feature is well depicted by the corresponding anomalies of vertically integrated moisture flux (vector, Fig. 3a), which indicate a strong decline in the climatological water vapor transport (shading, Fig. 3a). With the climatological return of northeasterlies over the South China Sea by September, the anomalous cyclonic circulation intensified therein, thus enhancing the climatological northerly flux component before penetrating inland and causing an effective reduction in both water supply to and convergence of water in SW China (Fig. 3b). The intensity of the cyclonic(anticyclonic) anomalous circulation over the South China Sea (northern China) was more pronounced in late (early) summer 

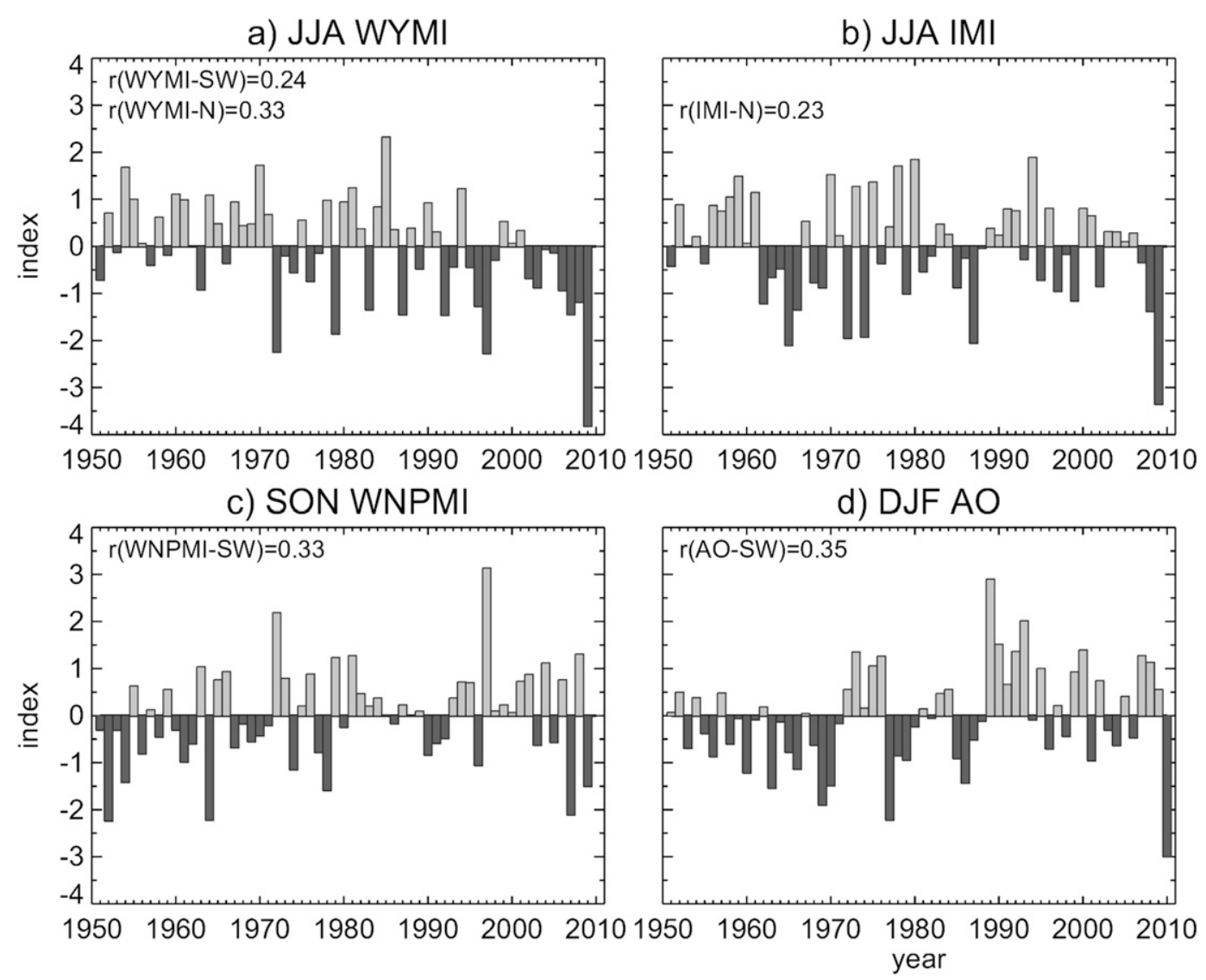

FIG. 4. Standardized time series of (a) summer WYMI (1951-2009), (b) summer IMI (1951-2009), (c) autumn WNPMI (1951-2009), d) winter AO (1951-2010), and their correlation coefficient with regional seasonal precipitation in $\mathrm{N}$ and SW China. Only correlations significant at $p<0.1$ level are shown. For simplicity, the seasonal times series are only shown until 2009 in (a), (b), and (c), and 2010 in (d), so the last bar in the series identifies the relevant value of the index during the drought episode.

and autumn, which is concurrent with the maximum precipitation reductions in SW (N) China (Fig. 2).

Note that in Fig. 3a there is no clear evidence of the typical summer Pacific-Japan tripole height pattern over East Asia associated with the EASM. Instead, the strong weakening of low-level cross-equatorial gyre and associated monsoon westerlies over the western Indian Ocean and the Arabian Sea feature a weak IM. A correlation analysis further supports that summer rainfall variations in $\mathrm{N}$ and SW China do not fully reflect changes in EASM but rather in the broad South Asian monsoon, as measured by the WYMI (Fig. 4a). The WYMI for summer 2009 was -3.8 , standing as the lowest in record, which may partially explain the regional deficits of precipitation. The relatively weak SW China rainfall response to WYMI may reflect a combined influence of the WNPM and IM (e.g., Ding and Chan 2005), with the former (latter) exerting a stronger influence in late (early) summer and autumn. In fact, monthly SW China rainfall and the WNPMI display maximum correlations in autumn, when the 2009 WNPMI reached values of -1.5 (Fig. 4c).

Summer N China rainfall is also weakly correlated with the IMI (Fig. 4b), which reached -3.3 in summer 2009, standing as the lowest value on record since 1951. Several studies have reported that the summer diabatic forcing from the IM can excite a quasi-stationary upperlevel disturbance, triggering a barotropic Rossby wave train structure along the subtropical westerly jet ( $\mathrm{Wu}$ et al. 2003; Sato and Takahashi 2006). This waveguide with anomaly cells of alternating signs, also called the Silk Road or Circumglobal Teleconnection pattern (CGT; Ding and Wang 2005), has one of its loading centers over northern China, thus affecting moisture transport to East Asia from the Indian Ocean. The anomalous upper-level height signature of Fig. 3a shows some resemblance with the CGT pattern and it provides a feasible mechanism to explain the summer deficits in N China via the suppression of diabatic heating associated with the weak IM. This is supported by recent studies that have found 
that the CGT pattern explains a considerable fraction of interannual rainfall variability over northern China (Huang et al. 2010).

In winter, the climatological mean pattern is characterized by northerly winds associated with the Siberian High that affect $\mathrm{N}$ China before turning to easterlies over the South China Sea and converging leeward of the Tibetan Plateau with humid fluxes from the Bay of Bengal (e.g., Wang et al. 2009). The corresponding composite for the 2010 winter (Fig. 3c) reveals that the anomalous circulation in the south coast of Asia diverted water transport to the east before penetrating inland toward SW China. The anomalous southwesterlies in the southeast coast of China are in agreement with the occurrence of mature El Niño events (e.g., Zhang et al. 1997; Wang and Feng 2011), which is frequently associated with a weak EAWM $(r=-0.24 ; p<$ 0.1 ). However, the EAWMI only displayed values slightly below zero in $2010(-0.1)$, despite the occurrence of strong El Niño conditions $(\mathrm{EN} 3.4=1.5)$. Unlike subtropics, the intense cooling in northern China is opposite to that expected from a weak EAWM and such a lack of coherence between subtropics and extratropics might partially explain the neutral values of the EAWMI. On the other hand, the spatial pattern of temperature anomalies over China resembles that associated with negative phases of the AO (Thompson and Wallace 2000), which in the winter of 2009/2010 exhibited its lowest value $(-3.0)$ since the midtwentieth century (Fig. 4d; Cohen et al. 2010), likely being able to overwhelm any ENSO influence. Previous studies have reported significant links between the AO and temperature and precipitation in China through the modulation effect of the AO on the Siberian High (e.g., Gong and Wang 2003; B. Wu and J. Wang 2002) or the polar vortex (e.g., Wang and Chen 2010b). Our results reveal significant correlations between SW precipitation and the winter AO index (Fig. 4d). Unlike SW China, winter rainfall in the $\mathrm{N}$ region tends to reflect fluctuations in the EAWM $(r=-0.30 ; p<0.1)$, and it presented near-normal values in winter 2010, as the EAWMI did. The spatial-dependent response of the precipitation to the $\mathrm{AO}$ is in agreement with Gong and Wang (2003), who found that the AO signal in precipitation is stronger in southern than in northern China, although the physical mechanisms are still poorly understood. A feasible explanation would involve dynamical responses resulting from the AO-related warming over the Tibetan Plateau. For example, Gao and Yang (2009) suggested that the thermally forced anticyclonic conditions arising from anomalous warming over the Tibetan Plateau can weaken the climatological India-Burma trough (represented by thick arrows in
Fig. 3c), thus suppressing moisture fluxes from the Bay of Bengal into mainland China.

\section{b. The possible influence of ENSO}

The summer of 2009 was characterized by an extremely anomalous situation, as featured by the IMI and WYMI as well as by maximum precipitation decreases in absolute terms over both $\mathrm{N}$ and SW China. On the other hand, the ENSO is well known to interact with the Asian monsoon subsystems and a developing phase of El Niño has been related with droughts in northern China (e.g., Huang et al. 2007). These results are suggestive of a possible ENSO influence on the summer drought that deserves further attention.

Our analyses do not reveal highly significant relationships between regional seasonal precipitation and (lead or lag) ENSO indices, with correlations being typically weaker than those found with the monsoon indices (not shown). Note, however, that these results do not exclude a possible indirect influence of ENSO. There are several reasons supporting this statement. First, the impacts of ENSO in China's summer precipitation are not opposite between developing and decaying phases of ENSO, so the ENSO-rainfall relationship cannot be fully reflected in linear correlation (e.g., Wallace et al. 1998; Wu et al. 2003). Second, the WYMI, by definition, is partially influenced by both the WNPM and the IM, which, in turn, are modulated by ENSO.

In particular, a weak (strong) WNPM tends to occur in El Niño (La Niña) developing years and La Niña (El Niño) decaying years (e.g., Wang et al. 2001), as was observed in summer 2009. This association indicates a plausible indirect path for ENSO affecting China's precipitation. However, as stated before, summer precipitation anomalies in $\mathrm{N}$ and SW China are not highly sensitive to the EASM, therefore masking any possible ENSO influence on the drought. On the other hand, the weak 2009 IM is also in agreement with the developing phase of El Niño (e.g., Wang et al. 2001). Nevertheless, recent studies have suggested a relative independence of the 2009 IM value in relation to ENSO. In particular, the outstanding 2009 summer Indian drought has been attributed to two pronounced breaks in (i) June, because of dry air mass intrusions associated with extratropical waves (Krishnamurti et al. 2010), and ii) July-August, because of internal (rather than ENSO forced) dynamics, associated with the interaction of the intraseasonal oscillation over IM and planetary-scale equatorial Rossby waves propagating westward as a remnant of the decaying Madden-Julian oscillation (Neena et al. 2011). This apparent lack of ENSO influence on the IM is also in agreement with the recent weakening of the IM and ENSO linkage, which has fallen to statistically 
a)
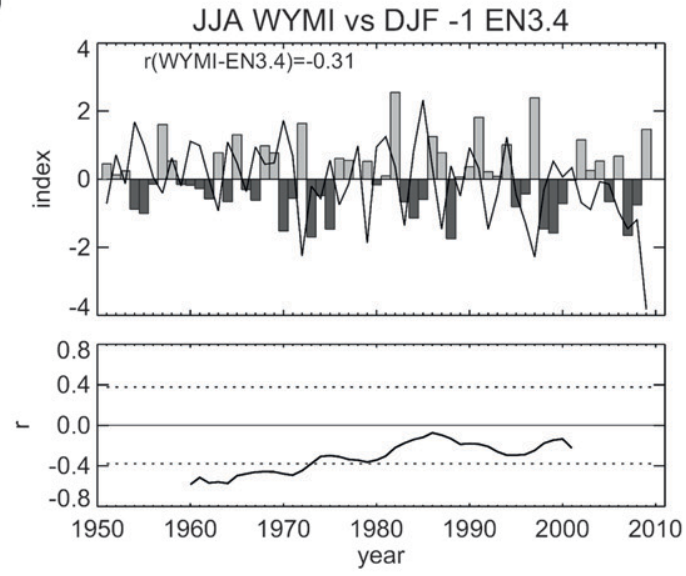

b)

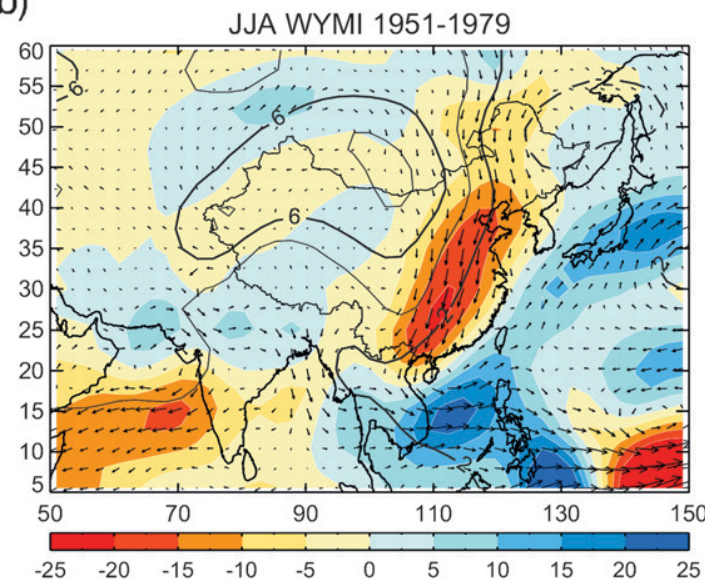

C)

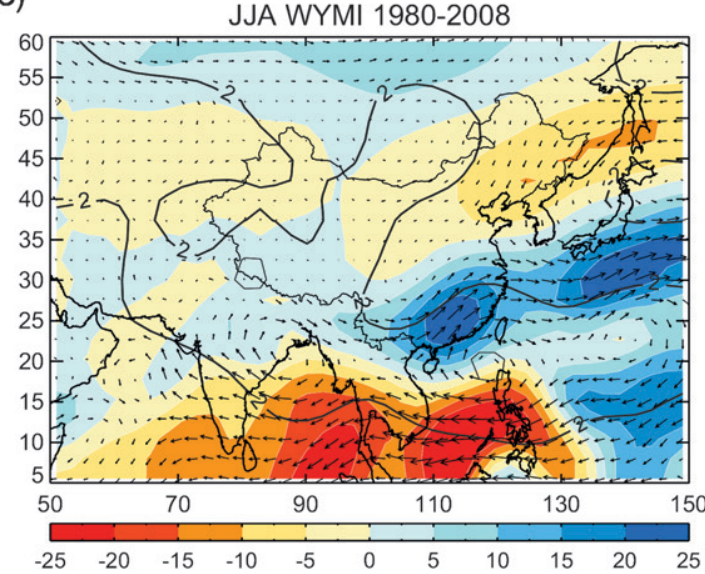

FIG. 5. (a) Time series of summer WYMI (solid line) and the following winter EN3.4 index (bars) and the 21-yr running correlation between them (bottom). Dashed lines indicate the statistical significance at $p<0.1$ level. The correlation for the full period of analysis is indicated at the top-left corner and it is significant at $p<0.1$. (b),(c) Regressed coefficients of geopotential height at $850 \mathrm{hPa}$ (gpm, contours), vertically integrated moisture flux from surface to $300 \mathrm{hPa}\left(\mathrm{kg} \mathrm{m}^{-1} \mathrm{~s}^{-1}\right.$, arrows), and total water transport (shading, $\mathrm{kg} \mathrm{m}^{-1} \mathrm{~s}^{-1}$ ) on the WYMI for the periods 1951-79 and 1980-2008, respectively. The regressed fields correspond to a standardized WYMI of -1 . nonsignificant levels in recent decades (e.g., Kumar et al. 1999). A similar weakening took place around the late 1970s between the WYMI and ENSO (Fig. 5a), suggesting that decadal variability of the WYMI is mainly controlled by the IM rather than by the WNPM.

To further explore the potential role of ENSO, we have regressed the WYMI onto several atmospheric fields for two different periods: (i) 1951-79 (Fig. 5b), characteristic of a strong WYMI-ENSO coupling (as shown in Fig. 5a), and ii) 1980-2008 (Fig. 5c), which features a weak WYMI-ENSO coupling (as shown in Fig. 5a). The former is considered to represent atmospheric circulation anomalies associated with the WYMI for those periods in which ENSO would have affected significantly the WYMI. The latter can be regarded to capture the WYMI features for periods of much weaker ENSO influence. Interestingly, the spatial pattern of anomalies associated with weak phases of the WYMI during the strong ENSO coupling period shows much better resemblance with the situation observed in summer 2009 (Fig. 3a) than the corresponding WYMI signatures for the most recent uncoupled period. The weakening of the land-sea pressure contrast and the corresponding suppression of southerly monsoon fluxes from southern to northern China are particularly noticeable in Figs. 3a and $5 \mathrm{~b}$. While no definitive conclusions can be drawn from this simple analysis, it indicates that the 2009 summer circulation was more prone to exhibit typical signatures of the summers with ENSO influence. These results are not in agreement with those found by Neena et al. (2011). On the other hand, Feng and $\mathrm{Hu}$ (2004) reported an ENSO signal in northern China summer precipitation only when ENSO and IM were coupled. According to these authors, the interactive linkage between the ENSO and summer precipitation in $\mathrm{N}$ China was activated by the IM through a moisture convoy emanating from the Indian Ocean and with signatures similar to the water flux anomalies of Fig. 3a. All these results indicate that while an ENSO influence on the drought through the WNPM is not fully evidenced from our analyses, a possible indirect ENSO influence via the IM should at least not be ruled out.

\section{Socioeconomic impacts}

\section{a. Drought impact on hydroelectrical production}

As stated in the introduction, SW China represents a major water reservoir of the country. This region supplies water to the head of Pearl and Yangtze Rivers and concentrates around the $70 \%$ of the hydroelectric plants in the country (Wang and Ma 2005). In this section we assess the consequences of the prolonged SW China 
drought in water reservoirs by analyzing monthly hydroelectrical power production (2003-10) provided by the China National Knowledge Infrastructure (CNKI; http://www.cnki.net).

Figure 6 a shows the national hydroelectrical power output from January 2003 to August 2010 in China. The hydroelectrical power output is characterized by a maximum during the wettest months of the year (Fig. 6a, blue line), which is in agreement with the seasonal cycle in precipitation and river streamflow (Chen et al. 2009). Besides, the long-term time series also shows an upward trend and an increasing amplitude in the annual cycle, most likely reflecting efficiency improvements and the construction of additional dams or upgrade of older ones (e.g., the continuous implementation of new turbines to the Three Gorges Dam) rather than climatic signals. To disentangle the trend and the multiplicative seasonal components from the remaining variability we applied a logarithm transformation to the raw data, thus removing the multiplicative seasonal cycle (Shumway and Stoffer 2000), and the resulting series was subsequently detrended. The so-corrected series is shown in Fig. 6a (red line), with its deseasonalized departures (i.e., monthly anomalies relative to each calendar month) provided at the bottom. A decline in electricity output was evidenced from June 2009 to June 2010, with the period from October 2009 to April 2010 experiencing a power reduction of $\sim 20 \%$. The rapid response in hydroelectrical production to the lack of precipitation could be due to the strong contribution of the SW region to the nationwide power output plus a delayed response to the relatively dry conditions that already experienced this region and areas along the Yangtze River in the winter of 2009 (Gao and Yang 2009). A similar decline in power output was also observed in 2006/07, which was associated to drying conditions that affected the upper and medium reaches of the Yangtze River (Fig. 6b), thus confirming a good correspondence between hydroelectrical output and the lack of precipitation in the major river basins.

\section{b. Drought impact on vegetation}

To assess the impact of the drought conditions upon vegetation dynamics it must be taken into consideration the stark differences of land-cover type across China. The main land-cover types (Fig. 7a) correspond to deserts in northwest China; grasslands over Tibet and east of inner Mongolia; agriculture cover types in the northeast of China; the medium and lower reaches of the Yellow, Pearl, and Yangtze Rivers; and forests and shrubs in southern China. It must also be noted that the annual cycle of vegetative cycle peaks at different times of the year depending on the land-cover type. This seasonal dependence is shown in Fig. 7b, where the spatial a)
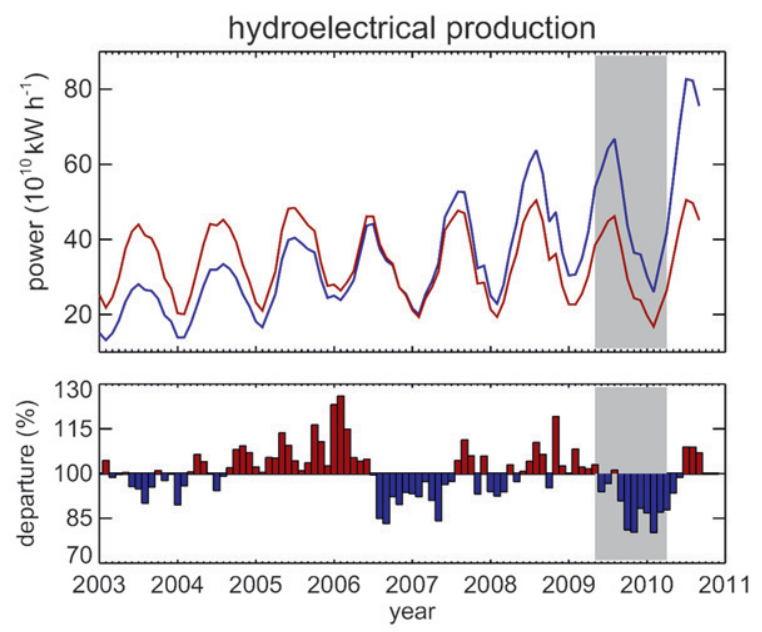

b)

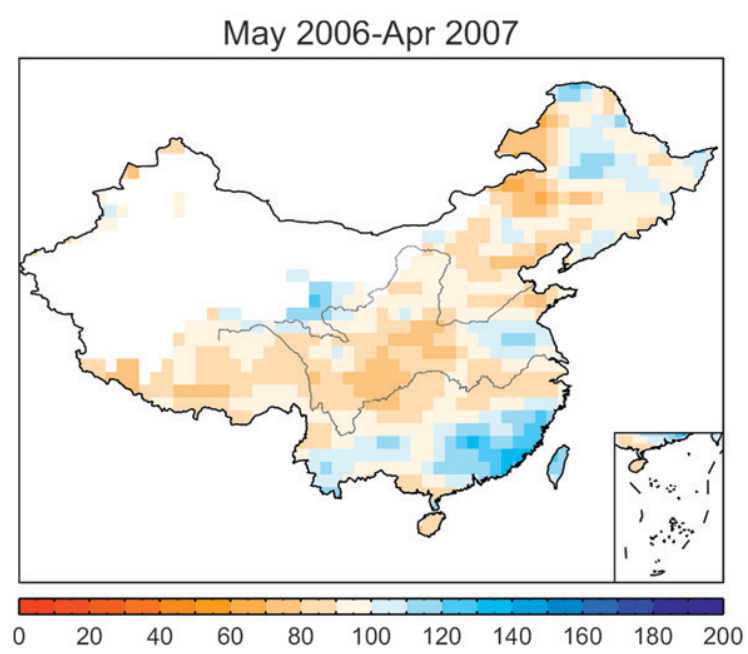

FIG. 6. (a) Monthly time series of hydroelectric power output $\left(10^{10} \mathrm{~kW} \mathrm{~h}^{-1}\right)$ in China for the period January 2003 to August 2010 (blue line). The red line shows the corrected time series after removing the multiplicative seasonality and linear trend. The bottom graphic shows the monthly anomalies (in percentage of the mean values for the period 2003-08). Gray shading identifies the drought episode (May 2009-April 2010). (b) As in Fig. 1 but for the period May 2006-April 2007.

distribution of the month corresponding to the maximum of the annual NDVI cycle is shown. Agriculture fields in the northern region mainly correspond to summer plantations, while vegetation greenness located in the south peaks in early summer (except those areas dominated by forests). On the other hand, the lowland regions located between the valleys of the Yellow and Yangtze Rivers display two annual NDVI maxima in early spring and late summer, corresponding to winter and summer plantations, respectively (e.g., Piao et al. 2010). The discrimination of these areas characterized by two harvests per year is further evidenced by a k-means cluster 


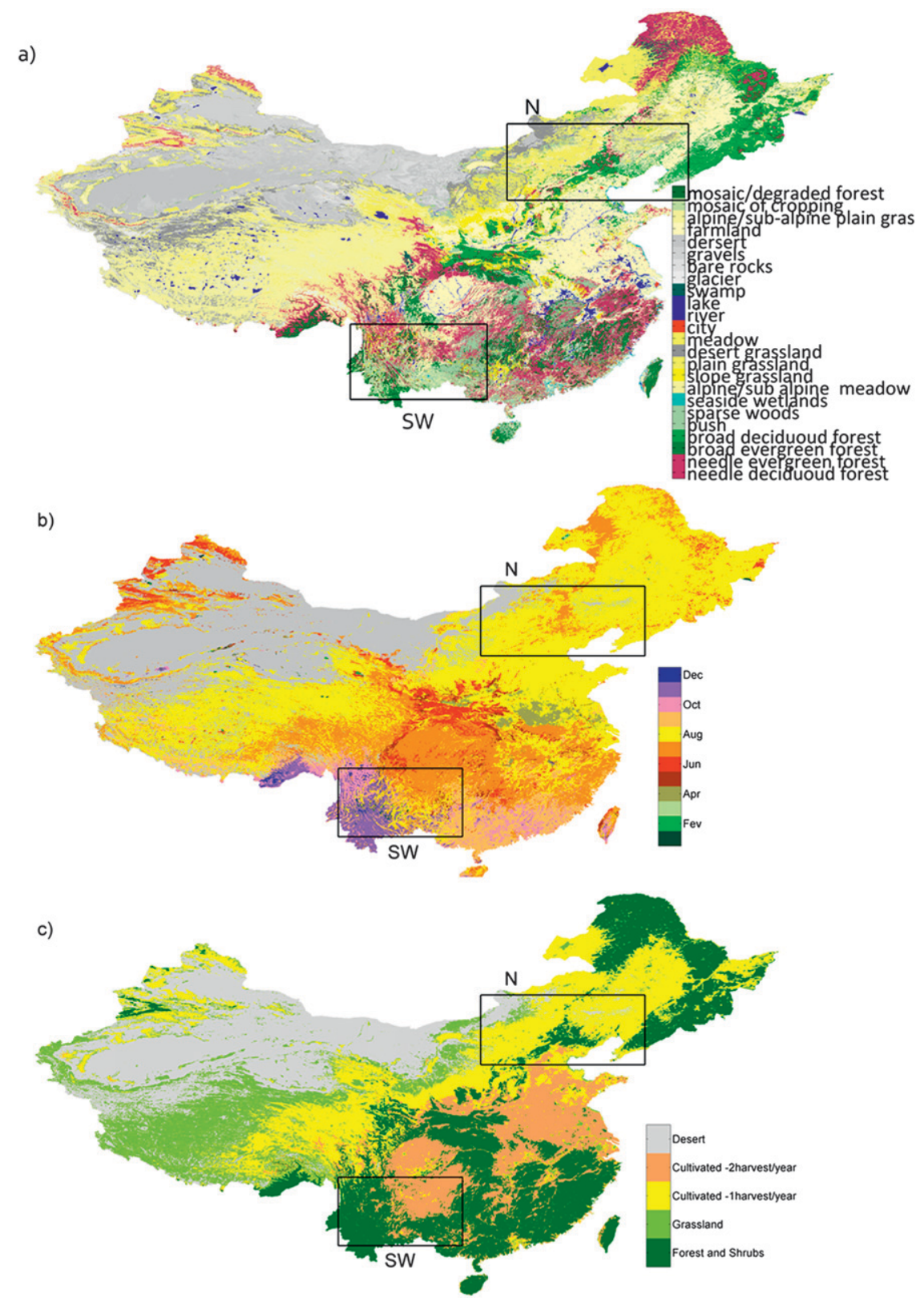

FIG. 7. (a) Land-cover classification as obtained from the Global Land Cover 2000 database; (b) spatial distribution of the month with maximum annual mean NDVI, as obtained from monthly average SPOT-VEGETATION data for the period 1998-2010; and (c) distribution of vegetation over China, according to the vegetative cycle of each pixel. The boxes labeled as $\mathrm{N}$ and SW indicate the major regions affected by the drought. The boxes are similar (but not equal) to the ones defined in Fig. 1. Pixels corresponding to bare soil and sparse vegetation have been masked and represented in light gray.

analysis applied to the monthly mean NDVI at each pixel. A total of five clusters (Fig. 7c) were predefined since no major discrepancies were found if five or more clusters were initially chosen.

We have evaluated the accumulated drought effects in vegetation by counting, for each pixel, the number of months with stressed vegetation between May 2009 and June 2010 (Fig. 8). The choice of this period takes into account that the annual cycle of vegetation dynamics approximately follows that of precipitation with a 0-2month delay. Besides, it includes the typical growing season (i.e., April-October) over most arable lands of 


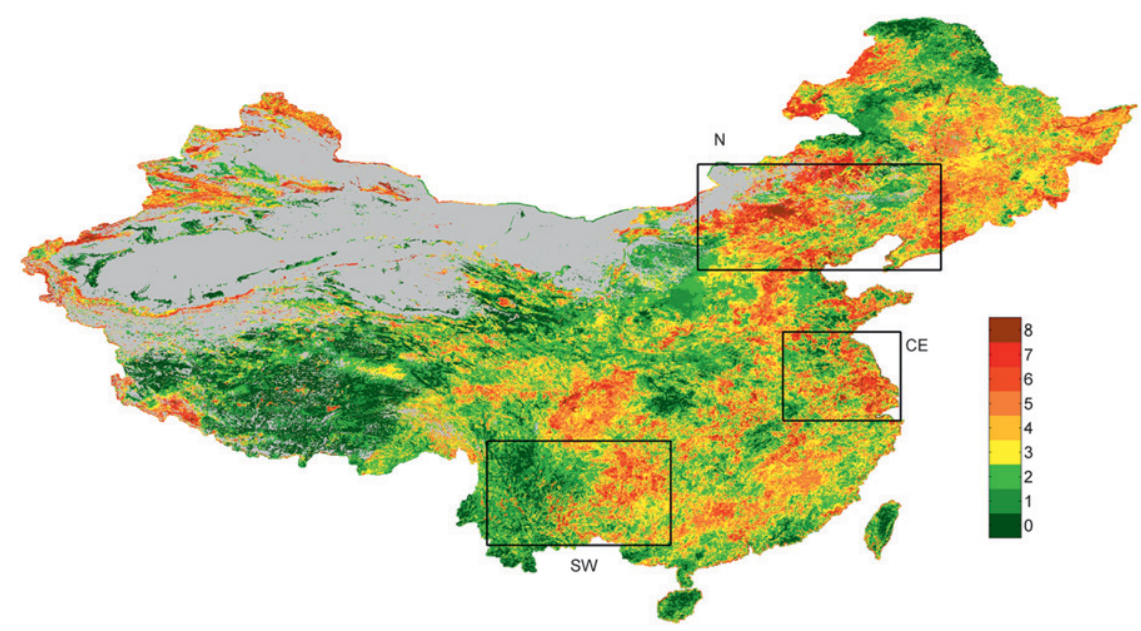

\begin{abstract}
FIG. 8. Number of months between May 2009 and June 2010 with weighted NDVI anomaly values below -0.10 . Pixels corresponding to bare soil and sparse vegetation have been masked and represented in light gray. The boxes labeled as N, and SW correspond to those shown in Fig. 7. The box labeled as CE highlights a third region with stressed vegetation.
\end{abstract}

China but also the growing season of winter wheat that spans October-April in many provinces of eastern China (e.g., Tao et al. 2008). Because of the strong regional differences in NDVI, monthly NDVI anomalies were weighted by the local mean of the analyzed period (19982010) and referred to as weighted NDVI anomalies from now on. A pixel is said to be under stress in a given month if the corresponding weighted NDVI is lower than a predefined threshold fixed to -0.10 . This threshold represents a compromise between more demanding values (that might miss months with important contribution to the annual vegetative cycle) and more relaxed thresholds (that would in fact reflect values near climatology). Nevertheless, no major differences in the results were found when applying different threshold values ranging between -0.10 and 0.20 . Additionally, the usage of the fixed threshold of -0.10 instead of using all negative anomalies (i.e., a threshold of 0 ) in our analysis allows disentangling a relatively long period of vegetation stress from more irrelevant periods, as those formed by a single month with lower-than-usual vegetation greenness.

The results reveal that the most-affected regions (up to 8 months of stressed vegetation) corresponded broadly to areas of N China, the NDVI responses being less intense (up to 6 months of stress) over southern areas of China (Fig. 8). The analysis of land-cover types suffering from more than 4 months of stress within May 2009-June 2010 indicates that 32\% (12\%) of the affected pixels inside the $\mathrm{N}$ box of Fig. 8 corresponded to farmlands (broadleaved deciduous forest). Conversely, $29 \%(20 \%)$ of the pixels affected by drought in the SW box corresponded to needleleaved evergreen forest (bush), whereas only $15 \%$ belonged to farmlands. The prolonged vegetative stress in $\mathrm{N}$ China may well reflect the dominance of croplands being affected by the strong shortage of precipitation during the monsoon season of 2009 (Fig. 2b) and is probably enhanced by the subsequent recurrence of cold surges during the extended winter of 2009/10 (Fig. 3c). On the other hand, the comparatively reduced impact in SW and other regions of southern China is in agreement with the dominance of forest and shrubs therein, which are typically more resistant to drought events (e.g., Gouveia et al. 2009). Interestingly, cultivated-managed areas lying between the estuaries of the Yellow and Yangtze Rivers (box labeled as CE in Fig. 8) also experienced several months of persistently stressed vegetation, as discussed below.

To assess the drought impact in crop plantation, farmland pixels affected by more than 4 months of vegetation stress were selected for further analyses. The annual cycles of the spatially averaged farmland NDVI were computed for each year of the entire period (1998-2010) and for the three regions indicated in Fig. 8. The corresponding evolution of regional mean weighted NDVI anomalies during 2009 and 2010 is shown in Fig. 9 (bars). To better understand the regional differences, special attention must be placed in those months characterized by climatologically high photosynthetic activity within each area, since negative NDVI anomalies during the growing season are particularly symptomatic of processes damaging vegetation dynamics and crop growth. The climatological annual cycle of NDVI for farmland in the $\mathrm{N}$ region is more pronounced than in SW China (Fig. 9, dotted lines) and the maximum values occur in August (one month later than in SW), which means a 

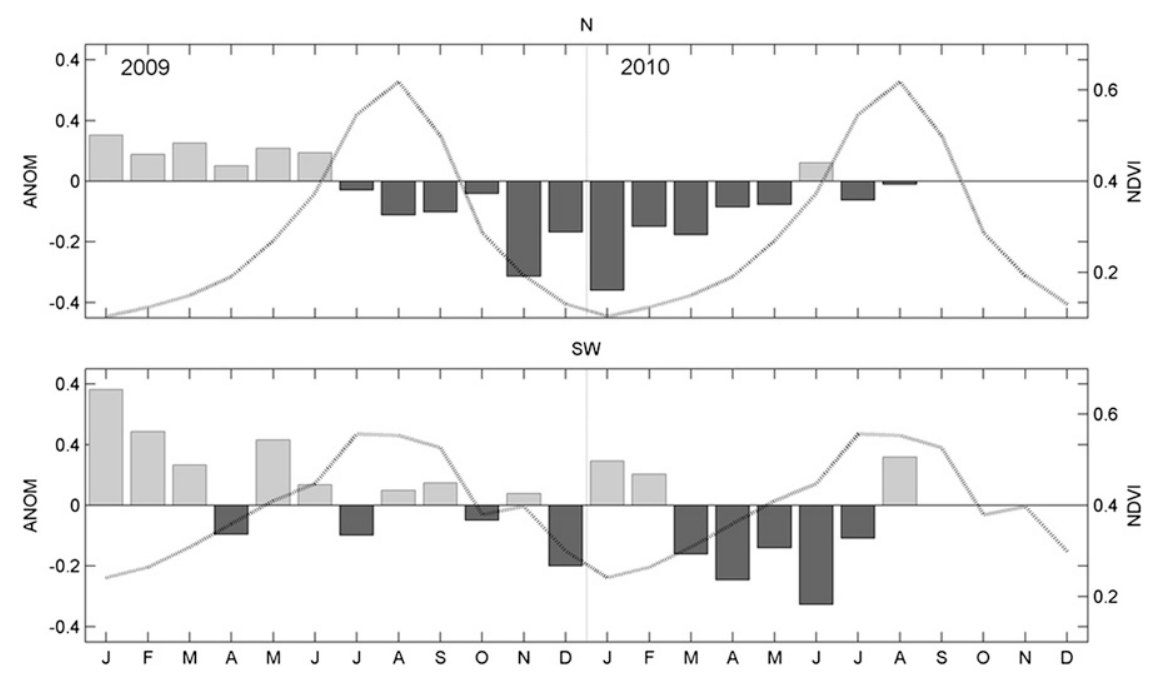

CE

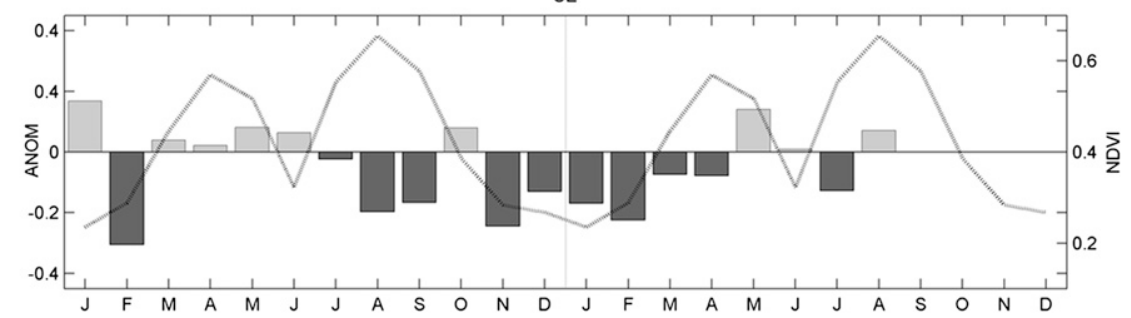

FIG. 9. Climatological (1998-2010) annual cycles of spatially averaged NDVI over farmland pixels affected by more than 4 months of vegetation stress (dotted line, right vertical axis) and the 2009/10 monthly time series of the weighted NDVI anomalies (bars, left vertical axis) averaged over (top) N, (middle) SW, and (bottom) CE China (see Fig. 8 for the definition of these regions).

delay of 1 month with the corresponding precipitation maximum (Fig. 2).

As discussed in section 3, the drought episode in $\mathrm{N}$ (SW) China was characterized by stronger deficits of precipitation during the peak (post) monsoon season of 2009. Thus, while the deficit of precipitation in N China started during the growing season, the decline of precipitation only become uninterrupted over SW China at the time of maximum vegetation greenness. Taking into account the dependence of crops to growing season precipitation, the earlier deficit of summer precipitation in $\mathrm{N}$ China (as compared to its climatological maximum in vegetative activity) would explain the negative impacts in summer farmlands vegetation greenness of this area. The persistence of negative NDVI anomalies in N China from July 2009 to May 2010 (Fig. 9a) occurs in tandem with those of the accumulated precipitation (Fig. 2a) and corroborates the sensitivity of this region to monsoon precipitation. On the other hand, the late summer deficit of precipitation in SW China may have not arrived on time so as to have a serious impact in summer 2009 crop growth. However, successive months with lack of precipitation resulted in pronounced anomalies in the accumulated precipitation totals (Fig. 2a), thereby affecting the growing season of 2010 crops (Fig. 9b) by presumably reducing soil moisture and impeding the germination of seeds.

Unexpectedly, some regions of eastern China (e.g., CE box in Fig. 8) also suffered from stressed vegetation, in spite of registering near-normal values in the May 2009April 2010 accumulated precipitation values (Fig. 1). The negative anomalies of farmland NDVI mostly span from the summer of 2009 until the spring of 2010, with the highest magnitude of vegetative stress occurring during the summer culture of 2009 (Fig. 9c). Recall that the farmland annual vegetative cycle for CE China is characterized by two annual maxima (Fig. 9c), the most prominent one occurring in August and a secondary one in April, which reflect the vegetative cycle of summer plantations (rice, maize, etc.) and winter wheat, respectively (e.g., Tao et al. 2008). Therefore, several factors may explain the negative NDVI anomalies over CE China. First, as these areas practice two cultures per year, seasonal precipitation is even more critical than accumulated totals. Consequently, the negative NDVI values during 2010 may have partially occurred in 
response to the precipitation deficits that affected this region in winter and spring of 2010 (section 3; note that these deficits are masked in the accumulated totals of Fig. 1 by the near-normal monsoon rains of 2009). Second, the fertile regions of the Yangtze and Yellow Rivers follow irrigation cultures, mainly in winter, and hence they are strongly dependent on water reservoirs. A considerable reduction in streamflow and water levels in damns (as evidenced between late spring of 2009 and early summer of 2010) would have caused negative impacts on both (i) summer plantations (mostly rice, which germinate in saturated soils and temporarily flooded estuaries of the rivers) due to the low streamflow reaching the lower catchments of major rivers and (ii) winter wheat crops by the lack of irrigation.

\section{Conclusions}

In this study we have described the recent 2009/10 drought episode over China, focusing on the temporal and spatial amplitude of the event, the associated atmospheric circulation anomalies, and the impact on the vegetation dynamics and hydroelectrical production of the country. While droughts are a relatively frequent phenomenon in northern China, the recent 2009/10 episode was particularly interesting because the suppression of regional rainfall $(\sim 25 \%$ below the average precipitation in the hydrological year) strongly affected SW China-a region that usually receives ample precipitation and accounts for much of the water reservoirs of the country. The peculiarity of this event also provides a valuable case study to highlight how responses in vegetative activity depend on not only the land-cover type and regional precipitation and temperature but also on the specific culture practices and management of water resources.

The spatiotemporal analysis of the precipitation showed that the 2009/10 drought episode corresponded to the driest hydrological year in SW China since 1951 and the second driest one in N China, herein falling relatively close to the recent major droughts that have struck this area (Wang et al. 2011). Our results stress that the precipitation regime in the $\mathrm{N}$ region is particularly sensitive to summer rainfall; hence, interdecadal or long-term changes on the physical driving mechanisms responsible for summer precipitation are prone to cause extremes and long-term trends in the annual precipitation totals. Conversely, the persistent drought in SW China was rather caused by an unusual sequence of dry months persisting between the extended summer 2009 and winter 2010 seasons.

An analysis of the associated large-scale atmospheric circulation suggests that the drought episode was at least partially influenced by large-scale processes at subtropical and extratropical latitudes-namely a weak large-scale South Asian monsoon in the summer season of 2009 (affecting both N and SW China) and a recordbreaking negative phase of the AO during winter 2010 (only for SW China). Although these results are supported by previous studies, the contribution of regional (e.g., Tibetan Plateau diabatic forcing), local (convective), and intraseasonal processes are also expected to have played a significant role in inhibiting the mechanisms conductive to regional precipitation. Moreover, our results suggest that summer precipitation in these regions is at least partially influenced by the broad-scale South Asian monsoon, while the EASM (mei-yu activity) may play a secondary role. More specifically, and in agreement with previous studies, $\mathrm{N}$ China summer precipitation seems to respond to the IM, while precipitation anomalies in SW China are also affected by the WNPM. Although a clear ENSO signal on the drought is not fully evidenced, the anomalously weak monsoon subsystems were in agreement with the developing El Niño phase, and the observed circulation features show good resemblance with periods of strong IM-ENSO coupling. However, the relatively low correlations between monsoon and ENSO indices and summer rainfall in $\mathrm{N}$ and SW China most likely reflect the influence of a large number of moisture sources (see Gimeno et al. 2010), interactions between the monsoon subsystems, intraseasonal oscillations, and a nonstationary ENSO signal (e.g., Feng and Hu 2004). Modeling studies of the 2009/10 drought are expected to bring a more objective evaluation of the different contribution of all monsoon systems and ENSO as well as other potential factors such as snow cover.

The evaluation of the accumulated number of months with negative NDVI values during May 2009 and June 2010 also reveals that the most-affected region corresponded to $\mathrm{N}$ China, which presented up to 8 months of persistently stressed vegetation. This is explained in terms of a dominance of monoculture farmlands in this region, their dependence on accumulated rainfall, and the dependence of the latter on summer precipitation, which was strongly reduced in 2009 over N China. On the other hand, this feature could also be related with a general grassland degradation trend observed from 1998 to 2007 over northern China (Zhao et al. 2012). The impact of the drought on vegetation was less persistent over southern China (including SW) because of the predominance of unmanaged land-cover types (forests and shrubs) that are particularly resistant to water shortage. However, the prolonged drought in this relatively small region of China was most likely responsible for a decline of $\sim 20 \%$ in nationwide hydroelectric output between autumn 2009 and spring 2010. Some areas of eastern China 
extending between the lower reaches of the Yangtze and Yellow Rivers also presented up to 6 months of persistently stressed vegetation. The distinctive vegetation responses across China stress the regional dependence on seasonal precipitation and water resources depending on the specific culture practices and land-cover types. Thus, the negative impacts on the valleys of the major rivers, where the accumulated precipitation did not show remarkable deficiencies, is well explained by the dominance of multicropping rotations within the year, water intensive crops, and their stronger sensitivity to precipitation in other seasons than summer. The reduction of storage water in damns was also potentially responsible for negative responses in downstream croplands of the Yangtze and Pearl Rivers.

The 2009/10 drought event raises concerns about the unequal distribution of water resources in China and its limited availability. Thus, despite that future Intergovernmental Panel on Climate Change (IPCC) projections in the region show large uncertainty and the recent agricultural benefits delivered by technical progress have so far offset the modest climate changes (Piao et al. 2010), the faster-growing population, industrial development, and intensive agricultural practices are likely to raise the competing demand of water resources, pointing to potentially serious vulnerabilities in China's future sustainability.

Acknowledgments. This study received support from the EU 7th Framework Program (FUME) Contract Number 243888 and from Portuguese Science Foundation (FCT) through the ENAC PTDC/AAC-CLI/103567/ 2008 project. LW is supported jointly by the Chinese Academy of Sciences (Grant KZCX2-EW-QN204) and the National Natural Science Foundation of China (Grant 41025017). We thank J. L. Chen for calculating the water vapor flux and J. Cao for helpful discussions. The NDVI dataset was kindly supplied by VITO database (http://free. vgt.vito.be), USDA/FAS/OGA and NASA Global Agriculture Monitoring (GLAM) Project. Precipitation data were provided by the GPCC (http://gpcc.dwd.de). The hydroelectrical power production data were provided by the China National Knowledge Infrastructure (CNKI; http://www.cnki.net). NCEP-NCAR reanalysis were supplied by the NOAA/OAR/ESRL PSD at http://www.esrl. noaa.gov/psd/. Two anonymous reviewers provided helpful comments that contributed to improve the manuscript.

\section{REFERENCES}

Chen, T.-C., S.-Y. Wang, W.-R. Huang, and M.-C. Yen, 2004: Variation of the East Asian summer monsoon rainfall. J. Climate, 17, 744-762.
Chen, W., S. Yang, and R.-H. Huang, 2005: Relationship between stationary planetary wave activity and the East Asian winter monsoon. J. Geophys. Res., 110, D14110, doi:10.1029/ 2004JD005669.

- L. Wang, Y. Xue, and S. Sun, 2009: Variabilities of the spring river runoff system in East China and their relations to precipitation and sea surface temperature. Int. J. Climatol., 29, 1381-1394.

Cohen, J., J. Foster, M. Barlow, K. Saito, and J. Jones, 2010: Winter 2009-2010: A case study of an extreme Arctic Oscillation event. Geophys. Res. Lett., 37, L17707, doi:10.1029/2010GL044256.

Ding, Q., and B. Wang, 2005: Circumglobal teleconnection in the Northern Hemisphere summer. J. Climate, 18, 34833505.

Ding, Y. H., and J. C. L. Chan, 2005: The East Asian summer monsoon: An overview. Meteor. Atmos. Phys., 89, 117-142, doi:10.1007/s00703-005-0125-z.

Fang, J. Y., S. L. Piao, Z. Tang, C. Peng, and W. Ji, 2001: Interannual variability in net primary production and precipitation. Science, 293, 1723

- — C. B. Field, Y. Pan, Q. Guo, L. Zhou, C. Peng, and S. Tao, 2003: Increasing net primary production in China from 1982 to 1999. Front. Ecol. Environ., 1, 293-297.

Feng, S., and Q. Hu, 2004: Variations in the teleconnection of ENSO and summer rainfall in northern China: A role of the Indian summer monsoon. J. Climate, 17, 4871-4881.

Gao, H., and S. Yang, 2009: A severe drought event in northern China in winter 2008-2009 and the possible influences of La Niña and Tibetan Plateau. J. Geophys. Res., 114, D24104, doi:10.1029/2009JD012430.

García-Herrera, R., D. Paredes, R. M. Trigo, I. F. Trigo, E. Hernández, D. Barriopedro, and M. T. Mendes, 2007: The outstanding 2004-2005 drought in the Iberian Peninsula: The associated atmospheric circulation. J. Hydrometeor., 8, 483-498.

Gilabert, M., S. Gandia, and J. Melia, 1996: Analyses of spectralbiophysical relationships for a corn canopy. Remote Sens. Environ., 55, 11-20.

Gimeno, L., A. Drumond, R. Nieto, R. M. Trigo, and A. Stohl, 2010: On the origin of continental precipitation. Geophys. Res. Lett., 37, L13804, doi:10.1029/2010GL043712.

Gong, D., and S. Wang, 2003: Influence of Arctic Oscillation on winter climate over China. J. Geogr. Sci., 13, 208-216.

Gouveia, C., R. M. Trigo, C. C. DaCamara, R. Libonati, and J. M. C. Pereira, 2008: The North Atlantic Oscillation and European vegetation dynamics. Int. J. Climatol., 28, 1835-1847.

,-- , and — 2009: Drought and vegetation stress monitoring in Portugal using satellite data. Nat. Hazards Earth Syst. Sci., 9, 185-195.

Habib, A. S., X. G. J. Chen, H. Wang, and L. Zhang, 2009: Analysis of China Vegetation Dynamics Using NOAA-AVHRR Data from 1982 to 2001. Geo-Spat. Inf. Sci., 12, 146-153, doi:10.1007/ s11806-009-0003-9.

Hagolle, O., A. Lobo, P. Maisongrande, B. Duchemin, and A. De Pereira, 2005: Quality assessment and improvement of SPOT/ VEGETATION level temporally composited products of remotely sensed imagery by combination of VEGETATION 1 and 2 images. Remote Sens. Environ., 94, 172-186.

Hobbs, T., 1995: The use of NOAA-AVHRR NDVI data to assess herbage production in the arid rangelands of Central Australia. Int. J. Remote Sens., 16, 1289-1302.

Holben, B. N., 1986: Characteristics of maximum-value composite images from temporal AVHRR data. Int. J. Remote Sens., 7, $1417-1434$. 
Hsu, H.-H., and X. Liu, 2003: Relationship between the Tibetan Plateau heating and East Asian summer monsoon rainfall. Geophys. Res. Lett., 30, 2066, doi:10.1029/2003GL017909.

Hu, Z.-Z., S. Yang, and R. Wu, 2003: Long-term climate variations in China and global warming signals. J. Geophys. Res., 108, 4614, doi:10.1029/2003JD003651.

Huang, G., Y. Liu, and R. Huang, 2010: The interannual variability of summer rainfall in the arid and semiarid regions of northern China and its association with the Northern Hemisphere circumglobal teleconnection. Adv. Atmos. Sci., 28, 257-268, doi:10.1007/s00376-010-9225-x.

Huang, R., Q. Guo, and J. A. Sun, 2000: Seasonal Charts of Climate Disasters in China. Ocean Press, 190 pp.

_ J. Chen, and G. Huang, 2007: Characteristics and variations of the East Asian monsoon system and its impacts on climate disasters in China. Adv. Atmos. Sci., 24, 993-1023.

Jhun, J.-G., and E.-J. Lee, 2004: A new East Asian winter monsoon index and associated characteristics of the winter monsoon. J. Climate, 17, 711-726.

Ju, J.-H., E.-X. Zhao, and J.-M. Lu, 2006: Impacts of the onset of the southeast Asian summer monsoon on the beginning of the rainy season in Yunnan. J. Trop. Meteor., 12, 205-208.

Kistler, R., and Coauthors, 2001: The NCEP-NCAR 50-Year Reanalysis: Monthly means CD-ROM and documentation. Bull. Amer. Meteor. Soc., 82, 247-267.

Kogan, F. N., 1995: Application of vegetation index and brightness temperature for drought detection. Adv. Space Res., 11, 91-100.

Krishnamurti, T. N., A. Thomas, A. Simon, and V. Kumar, 2010: Desert air incursions, an overlooked aspect, for the dry spells of Indian summer monsoon. J. Atmos. Sci., 67, 3423-3441.

Kumar, K. K., B. Rajagopalan, and M. A. Cane, 1999: On the weakening relationship between the Indian Monsoon and ENSO. Science, 284, 2156-2159, doi:10.1126/science.284.5423. 2156.

Maisongrande, P., B. Duchemin, and G. Dedieu, 2004: VEGETATION/ SPOT-An operational mission for the Earth monitoring: Presentation of new standard products. Int. J. Remote Sens., 25, 9-14.

Nan, S., and J. Li, 2003: The relationship between the summer precipitation in the Yangtze River valley and the boreal spring Southern Hemisphere annular mode. Geophys. Res. Lett., 30, 2266, doi:10.1029/2003GL018381.

Neena, J. M., E. Suhas, and B. N. Goswami, 2011: Leading role of internal dynamics in the 2009 Indian summer monsoon drought. J. Geophys. Res., 116, D13103, doi:10.1029/2010JD015328.

Nemani, R. R., C. D. Keeling, H. Hashimoto, W. M. Jolly, S. C. Piper, C. J. Tucker, R. B. Myneni, and S. W. Running, 2003: Climate-driven increases in global terrestrial net primary production from 1982 to 1999. Science, 300, 1560-1563.

Piao, S., and Coauthors, 2010: The impacts of climate change on water resources and agriculture in China. Nature, 467, 43-51, doi:10.1038/nature09364.

Qiu, J., 2010: China drought highlights future climate threats. Nature, 465, 142-143, doi:10.1038/465142a.

Rudolf, B., and U. Schneider, 2005: Calculation of gridded precipitation data for the global land-surface using in-situ gauge observations. Proc. Second Workshop of the Int. Precipitation Working Group, Monterey, CA, EUMETSAT, 231-247.

Sato, N., and M. Takahashi, 2006: Dynamical processes related to the appearance of quasi-stationary waves on the subtropical jet in the midsummer Northern Hemisphere. J. Climate, 19, 1531-1544.
Shumway, R. H., and D. S. Stoffer, 2000: Time Series Analysis and Its Applications. Springer, 549 pp.

Song, L., A. J. Cannon, and P. H. Whitfield, 2007: Changes in seasonal patterns of temperature and precipitation in China during 1971-2000. Adv. Atmos. Sci., 24, 459-473.

Sung, M.-K., W.-T. Kwon, H.-J. Baek, K.-O. Boo, G.-H. Lim, and J.-S. Kug, 2006: A possible impact of the North Atlantic Oscillation on the east Asian summer monsoon precipitation. Geophys. Res. Lett., 33, L21713, doi:10.1029/ 2006 GL027253.

Tao, F., M. Yokozawa, J. Liu, and Z. Zhang, 2008: Climate-crop yield relationships at provincial scales in China and the impacts of recent climate trends. Climate Res., 38, 83-94.

Thompson, D. W. J., and J. M. Wallace, 2000: Annular modes in the extratropical circulation. Part I: Month-to-month variability. J. Climate, 13, 1000-1016.

Trenberth, K. E., and C. J. Guillemot, 1995: Evaluation of the global atmospheric moisture budget as seen from analyses. J. Climate, 8, 2255-2280.

Trigo, R. M., C. M. Gouveia, and D. Barriopedro, 2010: The intense 2007-2009 drought in the Fertile Crescent: Impacts and associated atmospheric circulation. Agric. For. Meteor., 150, 1245-1257.

Tucker, C., C. Justice, and S. Prince, 1986: Monitoring the grasslands of the Sahel 1984-1985. Int. J. Remote Sens., 7, 15711581.

Wallace, J. M., E. M. Rasmusson, T. P. Mitchell, V. E. Kousky, E. S. Sarachik, and H. von Storch, 1998: On the structure and evolution of ENSO-related climate variability in the tropical Pacific: Lessons from TOGA. J. Geophys. Res., 103 (C7), 14 241-14 260.

Wang, A., D. P. Lettenmaier, and J. Sheffield, 2011: Soil moisture drought in China, 1950-2006. J. Climate, 24, 3257-3271.

Wang, B., and Z. Fan, 1999: Choice of South Asian summer monsoon indices. Bull. Amer. Meteor. Soc., 80, 629-638.

—_, and LinHo, 2002: Rainy season of the Asian-Pacific summer monsoon. J. Climate, 15, 386-398.

- R. Wu, and K. Lau, 2001: Interannual variability of the Asian summer monsoon: Contrasts between the Indian and the western North Pacific-East Asian monsoons. J. Climate, 14, 4073-4090.

— Z. Zu, J. Li, J. Liu, C.-P. Chang, Y. Ding, and G. Wu, 2008: How to measure the strength of the East Asian summer monsoon. J. Climate, 21, 4449-4463.

Wang, L., and W. Chen, 2010a: How well do existing indices measure the strength of the East Asian winter monsoon? Adv. Atmos. Sci., 27, 855-870, doi:10.1007/s00376-009-9094-3.

$\longrightarrow$, and $-2010 \mathrm{~b}$ : Downward Arctic Oscillation signal associated with moderate weak stratospheric polar vortex and the cold December 2009. Geophys. Res. Lett., 37, L09707, doi:10.1029/2010GL042659.

_- , and J. Feng, 2011: Two major modes of the wintertime precipitation over China. Chin. J. Atmos. Sci., 35, 11051116.

_- W. Chen, W. Zhou, and R. Huang, 2009: Interannual variations of East Asian trough axis at $500 \mathrm{hPa}$ and its association with the East Asian winter monsoon pathway. J. Climate, 22, 600-614.

Wang, Y., and Z. L. Ma, 2005: Rich hydroelectric resources's exploitation in southwest of China and its economic development. Resour. Dev. Mark., 21, 25-27.

Webster, P. J., and S. Yang, 1992: Monsoon and ENSO: Selectively interactive systems. Quart. J. Roy. Meteor. Soc., 118, 877-926. 
Wu, B., and J. Wang, 2002: Winter Arctic oscillation, Siberian High and East Asian winter monsoon. Geophys. Res. Lett., 29, 1897, doi:10.1029/2002GL015373.

Wu, G., and Y. Zhang, 1998: Tibetan Plateau forcing and the timing of the monsoon onset over South Asia and the South China Sea. Mon. Wea. Rev., 126, 913-927.

Wu, R., and B. Wang, 2002: A contrast of the East Asian summer monsoon-ENSO relationship between 1962-77 and 1978-93. J. Climate, 15, 3266-3279.

— Z.Z.Z. Hu, and B. P. Kirtman, 2003: Evolution of ENSO-related rainfall anomalies in East Asia. J. Climate, 16, 3742-3758.

Zhang, Y., K. R. Sperber, and J. S. Boyle, 1997: Climatology and interannual variation of the East Asian winter monsoon: Results from the 1979-95 NCEP/NCAR reanalysis. Mon. Wea Rev., 125, 2605-2619.
— T. Li, and B. Wang, 2004: Decadal change of the spring snow depth over the Tibetan Plateau: The associated circulation and influence on the East Asian summer monsoon. J. Climate, 17, 2780-2793

Zhao, Y., C. He, and E. Q. Zhang, 2012: Monitoring vegetation dynamics by coupling linear trend analysis with change vector analysis: A case study in the Xilingol steppe in northern China. Int. J. Remote Sens., 33, 287-308.

Zhou, L., C. J. Tucker, R. K. Kaufmann, D. Slayback, N. Shabanov, and R. B. Myneni, 2001: Variations in northern vegetation activity inferred from satellite data of vegetation index during 1981 to 1999. J. Geophys. Res., 106 (D17), 20 069-20 083.

Zou, X., P. Zhai, and Q. Zhang, 2005: Variations in droughts over China: 1951-2003. Geophys. Res. Lett., 32, L04707, doi:10.1029/ 2004GL021853. 